\title{
A Review on the Properties and Uses of Paracetamol
}

\author{
Iwuozor Kingsley Ogemdi \\ Department of Pure and Industrial Chemistry, Nnamdi Azikiwe University, Awka, Nigeria
}

Email address:

Kingsleyiwuozor5@gmail.com

\section{To cite this article:}

Iwuozor Kingsley Ogemdi. A Review on the Properties and Uses of Paracetamol. International Journal of Pharmacy and Chemistry. Vol. 5, No. 3, 2019, pp. 31-35. doi: 10.11648/j.ijpc.20190503.12

Received: August 13, 2019; Accepted: September 6, 2019; Published: September 21, 2019

\begin{abstract}
N-acylated aromatic amines (those having an acyl group, RCO- substituted on nitrogen) are important in over-the-counter headache remedies. Over-the-counter drugs are those you may buy without a prescription. Paracetamol is virtually the sole survivor of the so-called "aniline derivatives" or "aniline analgesics" which are; acetanilide, phenacetin and paracetamol (acetaminophen). Phenacetin and paracetamol are both derivatives of acetanilide. Paracetamol which is also referred to as 4-hydroxyacetanilide or para-hydroxyacetanilide to an Industrial Chemist is an important end product as well as an important precursor used in the synthesis of some other Organic compounds. As an analgesic it has gained fame because it is readily available but then its adverse effect stands pronounced when it's not taken in its rightful dosage or when it is taken in addition to some other food or drug. This article talks about the historical changes this important compound has undergone till date, its preparation by the chemist, some reactions it undergoes in the presence of some other precursors, its Dozage, Its adverse effect as well as its usage. This is a very helpful drug which becomes a poison in the presence of other drugs such as warfarin and care should be taken when handling this drug.
\end{abstract}

Keywords: Phenacetin, Drug-Drug Interaction, Preparation, Naphthalene, Acetanilide, Paracetamol

\section{Introduction}

Acetanilide, phenacetin, and acetaminophen are mild analgesics and antipyretics and are important, along with aspirin, in many non-prescription drugs [1].

The discovery that acetanilide was an effective antipyretic came about by accident in 1886. At the University of Strassburg, Professor Kussmaul, of the Department of Internal Medicine, asked two assistants to give naphthalene as a treatment for intestinal worms. Cahn and Hepp, who had been testing naphthalene as a possible vermifuge (an agent that expels worms) by accident, mixed up a bottle of acetanilide and the bottle of naphthalene. The patient's worms didn't disappear but his fever did - dramatically. In another instance of serendipity, it was soon in production and remained in use for several years because it was so cheap to produce. However, it had a serious side effect involving the deactivation of some of the hemoglobin in red blood cells. However, restrictions have been placed on its use due to kidney damage in long-term users. The publication of Cahn and Hepp describing their experiments with acetanilide caught the attention of Carl Duisberg, director of research at the Bayer Company in
Germany. Duisberg was confronted with the problem of profitably getting rid of nearly 50 tons of p-aminophenol; a by-product from the synthesis of one of Bayer's other commercial products. He immediately saw the possibility of converting p-aminophenol to a compound similar in structure to acetanilide, by putting an acyl group on the nitrogen. It was then believed, however, that all compounds having a hydroxyl group on a benzene ring (that is, phenols) were toxic. Duisberg devised a scheme of structural modification of p-aminophenol to get the compound phenacetin. Phenacetin turned out to be a highly effective analgesic and antipyretic. A common form of combination pain reliever, called an APC tablet, was once available. An APC tablet contained Aspirin, Phenacetin, and Caffeine (hence, APC). Phenacetin is no longer used in commercial pain-relief preparations. It was later found that not all aromatic hydroxyl groups lead to toxic compounds, and today the compound acetaminophen is very widely used as an analgesic in place of phenacetin. 'Acetaminophen' (4-acetamidophenol) is sold as the over-the-counter analgesic "Tylenol".

Acetaminophen is a pain-relieving (analgesic) and fever-treating (antipyretic) medication that can be obtained over-the-counter. Pharmaceutically it is a different kind of 
medication than other over-the-counter medications, such as ibuprofen and naproxen, which are also used to treat pain and fever. Acetaminophen is widely used for pediatric and adult fever and pain and comes in pill, liquid, injectable, and rectal suppository forms. Outside of the United States and Canada, acetaminophen is primarily known as "paracetamol" and is used for the same reasons [1].

Acetanilide was serendipitously found to possess antipyretic activity and quickly introduced into medical practice under the name of antifebrin Cahn and Hepp, and was shown to possess analgesic as well as antipyretic activities. But it's unacceptable toxic effects; the most alarming being cyanosis due to methemoglobinemia, prompted the search for less toxic aniline derivatives. A number of compounds were tested. The most satisfactory came out to be phenacetin (acetophenetidin) and N-acetyl-p-aminophenol (acetaminophen, paracetamol). Paracetamol had been synthesized by Morse in 1878. Phenacetin and paracetamol were introduced into clinical use in 1887 by von Mering, who soon discarded paracetamol in favor of phenacetin, because he assumed that the latter was less toxic. He found $\mathrm{N}-(4-H y d r o x y p h e n y l)$ ethanamide (Paracetamol) to be an effective antipyretic and analgesic, but wrongly thought that it caused the same hemoglobin problem as acetanilide. It was not until the 1940s that paracetamol was reinvestigated after it was found present in patients dosed with phenacetin. In 1953 paracetamol was marketed by Sterling-Winthrop Co., and promoted as preferable to aspirin since it was safe to take for children and people with ulcers. However, it causes liver damage from chronic use [1].

Paracetamol is rapidly formed in the guts of people who take phenacetin. It is the major metabolite (decomposition product) and it is likely that the antipyretic and analgesic effects of phenacetin were in fact due to paracetamol. There are some suggestions that the toxic effects of phenacetin were due to a minor metabolite - the N-oxide. Phenacetin is metabolized to two compounds. One involves the removal of the ethyl $\left(\mathrm{CH}_{3} \mathrm{CH}_{2}-\right)$ substituent from oxygen. The second involves the replacement of the hydrogen atom on nitrogen by a hydroxyl (-OH) group. This type of compound is called a hydroxamic acid.

Hydroxamic acids bind strongly to metal ions; this action may contribute to the toxicity. Albeit in part overshadowed by aspirin, introduced into medicine by Dreser in 1899, phenacetin has known for many decades an extraordinary popularity and has been indiscriminately used, especially as an ingredient of proprietary analgesic mixtures (particularly over-the-counter "headache mixtures," usually containing phenacetin, an aminopyrine derivative or aspirin, caffeine, and sometimes a barbiturate) and widely advertised to the public. The chronic overuse abuse of such mixtures by the laity, sometimes in prodigious amounts over periods of years, caused many serious chronic intoxications characterized by anemia, methemoglobinemia, and severe renal damage, with a high incidence of papillary necrosis ("analgesic nephropathy," "phenacetin nephropathy"). In 1948, Brodie and Axelrod demonstrated that the major metabolite responsible for the analgesic action of acetanilide and phenacetin is paracetamol, while methemoglobinemia is produced by another metabolite, phenyl hydroxylamine. So, paracetamol was "rediscovered" and marketed since the mid 1950s. It rapidly gained in popularity, and in many countries, including the United Kingdom; paracetamol sales exceeded those of aspirin since about 1980 . This was accompanied by the virtual commercial demise of phenacetin, blamed as the cause of "analgesic nephropathy," hematological toxicity, and psychotropic effects which may contribute to its liability for abuse [2].

Table 1. Table showing the Physical attributes of 4-hydoxyacetanilide.

\begin{tabular}{ll}
\hline Alternate names & Acetaminophen, p-Hydroxyacetanilide, p-acetyl aminophenol, Acamol, Abensanil \\
\hline Chemical formula & $\mathrm{C}_{8} \mathrm{H}_{9} \mathrm{NO}_{2}$ \\
Appearance & White odourless crystalline powder; large monoclinic prisms from water \\
Molecular weight & 151.16 \\
Melting point & $169-170.5^{\circ} \mathrm{C}$ \\
$\mathrm{Ph}$ & 5.3 to 6.5 at $25^{\circ} \mathrm{C}$. \\
Density & $1.293 \mathrm{gm} / \mathrm{cc}$ \\
& Soluble in water $\left(1: 70,1: 20\right.$ at $\left.100^{\circ} \mathrm{C}\right)$, ethanol $(1: 7)$, acetone $(1: 13)$, chloroform $(1: 50)$, glycerol $(1: 40)$, methanol $(1: 10)$, \\
Solubility & propylene glycol $(1: 9)$ and solutions of alkali hydroxides; insoluble in diethylether Slightly soluble in ether. It is insoluble \\
& petroleum ethers, pentone, and benzene. \\
Stability & Dry, pure paracetamol is stable to $45^{\circ} \mathrm{C}$ \\
Dissociation constant & $\mathrm{pKa}=9.0-9.5$ \\
Partition coefficient & Pc $=6.237$ (octanol: $\mathrm{pH} 7.2$ buffer) \\
\hline
\end{tabular}

This article is aimed at revealing the analgesic-Paracetamol with respect to an Organic Chemist view.

\section{Chemical Properties of Paracetamol}

\subsection{Preparation of Paracetamol}

\subsubsection{Phenol Formation}

Acetaminophen (also known as paracetamol) is a widely used analgesic. A route to acetaminophen involves three steps starting from phenol.
First, phenol is converted to nitro phenol via electrophilic aromatic substitution.

The nitro group of the Para-substituted nitrophenol is reduced to an amine either by sodiumborohydride $\left(\mathrm{NaBH}_{4}\right)$ reduction or direct hydrogenation.

Finally, the para-aminophenol is converted to acetaminophen via a reaction with acetic anhydride. Similar to an esterification of an alcohol through its reaction with a 
carboxylic acid, the nucleophilic amine attacks the extremely electrophilic carbonyl of the acetic anhydride.

Because the high reactivity of anhydrides and due to the low nucleophilicity of the carboxylic acid bi-product the formation of an amide via reaction with an anhydride has a much more favorable equilibrium constant as compared to the esterification of an alcohol by a carboxylic acid [3].

\subsubsection{Nitrobenzene Formation}

Nitrobenzene on being subjected to electrolytic reduction in the presence of sulphuric acid yields para-aminophenol which on treatment with acetic anhydride and sodium acetate gives rise to the production of acetaminophen (or paracetamol) [3].

\subsubsection{Para NitroChloroBenzene Formation}

The process by PNCB (Para nitrochlorobenzene) route is briefly described below.

Para nitro chloro benzene is reacted with caustic soda under pressure of $5 \mathrm{~kg} / \mathrm{sq} . \mathrm{cm}$ and temperature of $150^{\circ} \mathrm{C}$ for a period of 8 hours in an autoclave. The product of the reaction would be Para nitro phenol (PNP), which would be separated by crystallization and filtration.

The PNP would be treated with acetic acid to $\mathrm{pH}$ level of 3 and then subjected to reduction, for conversion of PNP to para amino phenol (PAP).

The Para amino phenol would be acetylated to produce crude Paracetamol.

The product would be further bleached with Activated carbon to produce Paracetamol of snow white colour. The product would be then dried in a tray dryer and then ground to the size of 40 microns [3].

\subsection{Structure Elucidation}

Paracetamol (p-Hydroxyacetanilide) undergoes usual reaction of Phenols because of the presence of its hydroxyl functional groups. It undergoes reactions such as acetylation, oxidation, reacts with sodium hydroxide and it gives a positive test to iron (iii) chloride test based on the presence of the phenolic group [4]. Other chemical reactions that they undergo include;

\subsubsection{Formation of 4-hydroxy-N-carboxylalanine}

When Paracetamol ( $\mathrm{p}$-Hydroxyacetanilide) is treated with acidic dichromate solution, the product formed (4-hydroxy-N-carboxylalanine) is formed in an oxidation reaction of the amide functional group [4]. The redox reaction for the reaction is given below:

\subsubsection{Formation of $\mathrm{N}$-acetyl-p-benzoquinoneimine (NAPQI)}

The toxic intermediate N-acetyl-p-benzoquinoneimine (NAPQI) is produced through the $\mathrm{N}$-oxidation of paracetamol to N-hydroxyparacetamol, followed by dehydration

\subsubsection{Titration with Ammonium Cerium (iv) Sulphate}

The British pharmacopoeia method for the analysis of paracetamol involves heating it under reflux with 1 moldm $^{-3}$ sulphuric acid. This is a straight forward, acid catalyzed, hydrolysis of an amine and a carboxylic acid. The 4-aminophenol which is formed is then titrated with an oxidizing agent, ammonium cerium (iv) sulphate using ferroin as the indicator [5].

The role of the ammonium cerium (iv) sulphate is to oxidize the 4-aminophenol to the iminoquinone. Only after all the 4-aminophenol has been oxidized will the cerium (iv) reagent oxidize the ferroin indicator from $\mathrm{Fe}^{2+}$ to $\mathrm{Fe}^{3+}$ (ferriin). During the titration the solution should be red, and the yellow end point is the transition from red to pale blue.

\subsection{Drug-Drug Interaction}

Paracetamol potentiates the anticoagulant effects of acenocoumarol and warfarin, with increased risk of bleeding. The suggested mechanisms are inhibition of the metabolism of oral anticoagulants; but more recent data did not confirm these hypotheses. Patients receiving oral anticoagulants should be cautioned to limit their intake of paracetamol. Carbamazepine increases the risk of Paracetamol hepatotoxicity by inducing the hepatic metabolism of paracetamol and thus increasing the formation of toxic metabolites Sulfinpyrazone, like carbamazepine, increases the risk of paracetamol toxicity by increasing the formation of hepatotoxic metabolites. Co-administration of paracetamol with zidovudine may result in neutropenia or hepatotoxicity; these effects were not been reported consistently [6].

Of major concern is the interaction with alcohol. Alcohol - paracetamol syndrome is defined as the development of acute toxic hepatic symptoms in long-term alcoholics who take paracetamol, in doses generally considered non-toxic. Patients with alcohol-paracetamol syndrome have a worse prognosis than non-alcoholic patients overdosed with paracetamol. Overall mortality in alcohol-paracetamol syndrome is about $20 \%$, and exceeds $75 \%$, if acute liver failure develops. Con-current use of alcohol and paracetamol may increase the $\mathrm{CYP}_{2} \mathrm{E}_{1}$-mediated metabolism of paracetamol to the highly hepatotoxic metabolite, $\mathrm{N}$-acetyl-p-benzoquinoneimine (NAPQI). In non-alcoholics, NAPQI is detoxified by conjugation with glutathione. In alcoholics, the combination of $\mathrm{CYP}_{2} \mathrm{E}_{1}$ induction and glutathione depletion results in NAPQI accumulation. In these subjects, the highest risk of paracetamol toxicity occurs after a brief (12h) abstinence of alcohol, since $\mathrm{CYP}_{2} \mathrm{E}_{1}$ is still induced, but alcohol is not present to compete for $\mathrm{CYP}_{2} \mathrm{E}_{1}$ metabolism. In addition, paracetamol has been shown to have lower bioavailability in epileptic patients receiving enzyme inducing anticonvulsants, including phenytoin and fos-phenytoin. On the other hand, paracetamol enhances the urinary elimination of lamotrigine.

\section{Dosage and Adverse Effect of Paracetamol}

\subsection{Dosage}

Although the maximum daily dosage has become 
somewhat controversial in recent years, it is generally recommended to follow the dosing instructions on the package of medications purchased over-the-counter. For children, dosing for most medications is based on the child's weight. The packaging may refer to dosing in terms of milligrams (mg) of medication per kilogram $(\mathrm{kg})$ of a child's weight. According to the Tylenol professional product monograph, "for adults and children 12 years of age and older, the recommended dose of acetaminophen is 650 to $1000 \mathrm{mg}$ every 4 to 6 hours as needed, not to exceed $4000 \mathrm{mg}$ in 24 hours... For children under 12 years of age, the recommended dose of acetaminophen is 10 to $15 \mathrm{mg} / \mathrm{kg}$ every 4 to 6 hours, not to exceed 5 doses (50 to $75 \mathrm{mg} / \mathrm{kg}$ ) in 24 hours." It should be noted that various concentrations of liquid acetaminophen for infants and children exist, so it is strongly recommended to read the dosing instructions very carefully and call your child's doctor with any questions about acetaminophen dosing $[7,8]$.

\subsection{Adverse Effect of Paracetamol}

With normal theraupeutic doses, paracetamol is virtually free of any significant adverse effects. Skin rash and other allergic reactions occur occasionally. The rash is usually erythematous or urticarial, but sometimes it is more serious and may be accompanied by drug fever and mucosal lesions. Patients who show hypersensitivity reactions to the salicylates only rarely exhibit sensitivity to paracetamol and related drugs There may be minor alterations in leukocyte count, but these are generally transient. In a few isolated cases, the use of paracetamol has been associated with agranulocytosis, neutropenia, thrombocytopenia, and pancytopenia. Paracetamol causes methaemoglobinemia and oxidative haemolysis in dogs, pigs and cats but not normally in humans, even after over dosage. Strain-dependent cataract formation and other ocular abnormalities have been described in induced mice and in one study, paracetamol produced a high incidence of liver cell tumors in $1 \mathrm{~F}$ mice. High doses of paracetamol given chronically to animals may cause testicular atrophy and inhibition of spermatogenesis [9].

The hepatoxicity of paracetamol is generally accepted by the formation of NAPQI, a metabolite formed by cytochrome $\mathrm{P} 450$. The quantitatively most significant of these is $\mathrm{CYP}_{2} \mathrm{E}_{1}$. A number of recent reports indicate that massive overdose of paracetamol can produce a fulminate acute centrilobular hepatic necrosis in humans and experimental animals.

In scenarios of Overdozage, There is a risk of poisoning, particularly in elderly subjects, in young children, in patients with liver disease, in cases of chronic alcoholism, in patients with chronic malnutrition and in patients receiving enzyme inducers. Poisoning may be fatal in these cases. Acute overdose with paracetamol may also lead to acute renal tubular necrosis.

Symptoms generally appear within the first 24 hours and comprise of nausea, vomiting, anorexia, pallor and abdominal pain. Overdose, $7.5 \mathrm{~g}$ or more of paracetamol in a single administration in adults or $140 \mathrm{mg} / \mathrm{kg}$ of body weight in a single administration in children, causes cytolytic hepatitis likely to induce complete and irreversible hepatic necrosis, resulting in acute or fulminant hepatic failure, hepatocellular insufficiency, metabolic acidosis and encephalopathy which may lead to coma and death [10].

\section{Paracetamol Usage}

Paracetamol lacks anti inflammatory action in rheumatic disorders. However, it is less toxic than Aspirin and does not produce anemia and liver damage, which sometimes result from the continued use of acetanilide and acetophenotidine.

Paracetamol is used in the treatment of Reduction of fever, Relief of muscle and joint \& pain, Relief of cold and flu symptoms, Relief of common headache.

\subsection{Fever and Body Temperature}

It is well known that paracetamol is antipyretic. It reduces fever in multiple species. A central site of antipyretic action against induced fever was demonstrated in rabbits by direct injection into the organum-vasculosum-lamina-terminalis (OVLT) located in the anterior wall of the third intra-cerebral ventricle. It is less well known that paracetamol can also lower a febrile body temperature. For example, several studies, employing different methods and routes of administration, have shown that paracetamol produces hypothermia in mice when the drug is administered intravenously (160 $\mathrm{mg} / \mathrm{kg}$, $2.5^{\circ} \mathrm{C}$ decrease), intraplantarily $\left(100-300 \mathrm{mg} / \mathrm{kg}\right.$ with $0.4-2{ }^{\circ} \mathrm{C}$ decrease respectively) or intra-cerebrovascularly (dose, $0.25^{\circ} \mathrm{C}$ decrease).

The data in humans are mixed. Effective and rapid reduction in brain temperature $\left(2^{\circ} \mathrm{C}\right)$ was reported with a single $1000 \mathrm{mg}$ dose of paracetamol in patients with subarachnoid haemorrhage or head trauma and oral or suppository paracetamol given $6 \mathrm{~g}$ daily to stroke patients lowered a febrile body temperature by $0.3^{\circ} \mathrm{C}$, a decrease attributed with reducing relative risk by $10-20 \%$. However, oral paracetamol $(650-1300 \mathrm{mg})$ was reported to not lower core body temperature in normothermic cardiac or stroke (3900mg daily) patients. Thus, paracetamol-induced hypothermia appears to be clinically insignificant when given at therapeutic daily dose $<4 \mathrm{~g}$.

\subsection{Inflammation}

Paracetamol has been reported to suppress various inflammation-related substances in animals and in inflamed dental tissue (1000 mg pretreatment and $4000 \mathrm{mg}$ post-surgery in patients with two-third molar extractions), but paracetamol is generally not considered to display very effective anti-inflammatory action in the clinical setting.

For example, paracetamol given i.p. or orally at $100 \mathrm{mg} / \mathrm{kg}$ (62), i.v. at $100-300 \mathrm{mg} / \mathrm{kg}$ or intrathecally at 200 micro g $\mathrm{kg}$ reduced inflammatory pain, but had no effect on edema and in a randomized, double-blind, placebo-controlled trial no significant improvement was seen in the paracetamol (1000mg four times daily) group when assessed 2 and 12 weeks into treatment. The relatively poor anti-inflammatory 
effect of paracetamol is a characteristic distinction from the NSAIDs and might be a reflection of different mechanism of action.

\subsection{Platelet Aggregation}

Because of the common impression that paracetamol lacks clinically relevant anti-platelet action, it is often used to avoid the bleeding risk associated with aspirin and other NSAIDs. There is some evidence of anti-platelet activity of paracetamol in human blood samples using in-vitro and ex-vivo assays, but other studies suggest a lack of anti-platelet action. Such an action, when present, is believed to be reversible (shorter acting), in contrast to the irreversible action of aspirin and NSAIDs. At least two recent clinical trials report that paracetamol did not interrupt platelet aggregation when given at $1000 \mathrm{mg}$ (73) or $3000 \mathrm{mg}$ i.v. Paracetamol might or might not interact with NSAIDs on this endpoint. The relatively poor inhibition of platelet aggregation by paracetamol is another characteristic distinction from the NSAIDs that might be a reflection of a different mechanism of action.

\section{Conclusion}

Various stories are heard about this very helpful at the same time deadly drug. While some appreciate it for its Relief of muscle and joint pain, cold and flu symptoms, common headache, antipyretic, anti-inflammatory functions, others curse it for its ability to lead to renal and hepatic complications in the human body. Paracetamol is one drug known and recognized by many but its chemistry is known by a select few. This article has brought to light the chemical properties of Paracetamol which can be used as a pre-cursor in the production of other chemical substances. One of its chemistry that should be taught to all is the drug interaction of this very powerful drug. In the presence of other drugs like warfarin, it causes excessive bleeding; patients should also stay away from Alcohol when taking this drug. Its adverse effects that results from overdozage should not be taken lightly as it can lead to range of sicknesses from skin rashes, vomiting to even a damaged liver or kidney, therefore the right dosage should be given to the patient and the patients should adhere to it.

\section{References}

[1] Iloamaeke, I. M., and Iwuozor, K. O. (2018). Quality assessment of selected Paracetamol tablets sold at Bridge Head Market, Onitsha, Anambra state, Nigeria. British Journal of Pharmaceutical and Medical Research, 3 (5): 1190-1197.

[2] B. Alfio, CNS drug reviews, 2006, 12 (3-4), 251-260.

[3] K. Ashutosh, New Age International (P) limited, Publishers, New delhi, 2004, 83-89.

[4] E. Frank, Royal Society of Chemistry, London, 2002, 1-13.

[5] IARC, IARC Monograph on Paracetamol (Acetaminophen): An Updating of IARC Monographs, 1990, 1-50, 307-309.

[6] M. Jones, and F. Carey, Norton publisher, London, 2005, 879-896.

[7] A. K. Pryzybyl, J. Kurek, Laboratory of Organic Chemistry Natural products and pharmaceuticals, 2013, 5-6.

[8] N. G. Swarooparani, International Journal of Chemical and Physical Sciences, 2015, 8 (4), 289-294.

[9] K. Toussaint, Journal of Clinical Pharmacy and Therapeutics, 2010, 4 (35), 620-626.

[10] Iwuozor, K. O (2018). Quality assessment of selected Vitamin C tablets sold at Bridge Head Market, Onitsha. Journal of Chemical and Biomolecular Engineering, 3 (3); 47-50.

[11] Jozwiak-Bebenista, M., and Nowak, J. Z. (2014). Paracetamol: Mechanism of Action, Applications and Safety Concern. Acta Poloniae Pharmaceutica-Drug Research, 71 (1); 11-23.

[12] Asare-Nkansah, S., and Kwakye, J. K. Quality Evaluation of paracetamol in the Bulk, Dosage Forms and Body Fluids using the High Performance Liquid Chromatography (HPLC) technique, 19-26.

[13] Mansour, O., Isbera, M., Mtaweg, A. (2017). Evaluation of Physical and Chemical Properties of Paracetamol (500mg) Tablets Marketed in Syria. Journal of Chemical and Pharmaceutical Sciences, 10 (3); 1309-1311.

[14] Srabovic, M., Huremovic, M., Catovic, B., Kulic, S., and Taletovic, A. (2017). Design synthesis and crystallization of acetaminophen, 7 (1); 218-230.

[15] Eynde, J. J. V. (2016). How Efficient is my (Medicinal) Chemistry? MDPI Journal of Pharmaceuticals, 9 (26); 1-16. 